\title{
EFEITO DA TEMPERATURA E DO TEOR DE GORDURA NA MASSA ESPECÍFICA DO LEITE DE COCO
}

César Augusto CANCIAM*

\author{
*Mestre em Engenharia Química, professor lotado no Departamento Acadêmico de Engenharia Química da \\ Universidade Tecnológica Federal do Paraná - Câmpus Ponta Grossa, canciam@utfpr.edu.br
}

Recebido em: 10/02/2015 - Aprovado em: 05/06/2015 - Disponibilizado em: 15/07/2015

\begin{abstract}
RESUMO:
A massa específica é um parâmetro físico-químico usado na caracterização dos materiais. O objetivo desse trabalho foi avaliar o efeito da temperatura e do teor de gordura sobre a massa específica do leite de coco. Para cada teor de gordura (de 20 a 35\%), foi encontrada uma relação do tipo Arrhenius. Os valores encontrados para a energia de ativação variaram de 463,9528 (teor de gordura de $20 \%$ ) a 467,7257 J.mol ${ }^{-1}$ (teor de gordura de $30 \%$ ), em um intervalo de temperatura entre 333,15 a $353,15 \mathrm{~K}$ e pressão de $1 \mathrm{~atm}$. Os resultados sugerem que com o aumento do teor de gordura, aumenta-se a energia de ativação, entre teores de gordura de 20 a 30\%. Nos teores de gordura de 30 a $35 \%$, ocorre uma diminuição da energia de ativação. Na literatura, não foram encontrados valores experimentais da energia de ativação para a massa específica do leite de coco, o que dificultou a comparação dos resultados.

PALAVRAS-CHAVE: Massa específica. Leite de coco. Efeito. Temperatura. Teor de gordura.
\end{abstract}

\section{Effect of temperature and fat content in the specific weight of coconut milk}

\begin{abstract}
:
The specific weight is a physic-chemical parameter used for characterization of materials. The objective of this work was evaluate the effect of temperature and fat content on specific weight of coconut milk. For each fat content, it was found an Arrhenius-type relationship. The values found for the activation energy ranged from 463.9528 (20\% fat

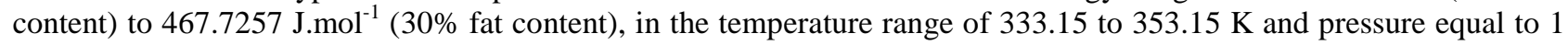
atm. The results suggest that increasing fat content, increases the activation energy, between fat contents of 20 to $30 \%$. In fat contents of 30 to $35 \%$, decreases the activation energy. In the literature, it was not found experimental values of activation energy for specific weight of coconut milk, making it difficult to compare the results.
\end{abstract}

KEYWORDS: Specific weight. Coconut milk. Effect. Temperature. Fat content.

\section{INTRODUÇÃO}

Massa específica, viscosidade, capacidade calorífica, condutividade térmica e difusividade térmica são consideradas como propriedades termofísicas dos materiais (MAGERROMOV et al., 2008).

O conhecimento do valor das propriedades termofísicas de um material é importante em muitas aplicações nas áreas da Ciência e da Engenharia, em particular nos cálculos relacionados à Termodinâmica (balanços energéticos e equilíbrio químico), na Mecânica dos Fluidos e na Transferência de calor e de massa subjacentes às Operações Unitárias (MATOS, 1998).

Equações do tipo Arrhenius permitem modelar matematicamente um determinado 
parâmetro físico-químico, bem como verificar o efeito da temperatura sobre esse parâmetro. É o que acontece com a viscosidade absoluta e a massa específica (TSEN e KING, 2002; GIAP, 2010). A Equação 1 expressa a massa específica em função da temperatura absoluta e corresponde a uma relação do tipo Arrhenius (TSEN e KING, 2002; CANCIAM, 2014):

$$
\rho=\rho_{\infty} \cdot \exp \left(\frac{E_{a}}{R \cdot T}\right)
$$

Em que $\rho$ corresponde à massa específica, $\rho_{\infty}$, à massa específica quando a temperatura tende ao infinito; $E_{a}$, à energia de ativação para a massa específica; $R$, à constante dos gases ideais e $T$ corresponde a temperatura absoluta.

Para a obtenção dos valores da energia de ativação para a massa específica $\left(E_{a}\right)$ e da massa específica quando a temperatura tende ao infinito $\left(\rho_{\infty}\right)$ pode ser empregado o método de linearização de curvas proposto por Freund (2004), na qual a Equação 1 é reescrita na forma de:

$$
\ln \rho=\ln \rho_{\infty}+\left(\frac{E_{a}}{R}\right) \cdot \frac{1}{T}
$$

O gráfico $\ln \rho$ versus $\frac{1}{T}$ fornece uma reta, em que o coeficiente angular corresponde à razão $\left(\frac{E_{a}}{R}\right)$ e o coeficiente linear, à $\ln \rho_{\infty}$ (CANCIAM, 2014).
Com relação à energia de ativação para a massa específica $\left(E_{a}\right)$, essa grandeza indica a sensibilidade da massa específica devido à variação da temperatura. Ou seja, para valores elevados da energia de ativação para a massa específica indicam uma mudança mais rápida na massa específica com a temperatura (CANCIAM, 2014).

O leite de coco pode ser definido como sendo uma emulsão do tipo óleo-água, obtida por trituração e prensagem do endosperma do coco, com ou sem adição de água. Essa emulsão é estabilizada por proteínas e provavelmente por alguns íons adsorvidos na interfase óleo-água. Sua composição é basicamente de água e gordura, porém contem pequenas quantidades de carboidratos, proteínas e minerais (TEIXEIRA et al., 1989; ALCÂNTARA et al., 2012).

De acordo com Schons (2008), o equilíbrio de uma emulsão pode ser compreendido através do balanço entre forças de atração e repulsão entre as partículas. A Teoria DLVO, elaborada pelos cientistas russos Derjaguin e Landau e pelos cientistas holandeses Verwey e Overbeek, sugere que a estabilidade de uma emulsão pode ser descrita com a interação entre suas forças atrativas e repulsivas. Essa interação ocorre através da aproximação das espécies químicas, chegando a chocarem-se entre si, devido ao movimento browniano. Dessa forma, ocorre atração 
devido às forças de Van der Waals e repulsão devido às forças eletrostáticas.

Teixeira, Coutinho e Gomes (2001) comentam que as forças de Van der Waals são originadas a partir da rápida flutuação da densidade eletrônica de cada espécie química, que induz a formação de um momento elétrico entre as espécies químicas vizinhas, fazendo com que essas se atraiam.

As forças de Van der Waals apresentam raio de ação curto, atuando unicamente entre regiões de uma e outra espécie química, que se encontram em contato mútuo, ou seja, as forças de Van der Waals atuam apenas entre as superfícies das espécies químicas (MORRISON e BOYD, 1996).

A massa específica de um líquido reflete o grau de empacotamento de suas espécies químicas, o que é decorrência da intensidade das interações entre as espécies. Assim, interações mais intensas tende a relacionar-se com massas específicas maiores. Como volume e massa específica são inversamente proporcionais. Então, quanto mais fortes as interações, mais empacotadas estão as espécies químicas, portanto o volume será menor e consequentemente a massa específica será maior (BROWN e HOLME, 2009).

O objetivo desse trabalho foi avaliar o efeito da temperatura e do teor de gordura sobre a massa específica do leite de coco. Para cada teor de gordura (de 20 a 35\%), foi encontrada uma relação do tipo Arrhenius e determinados os parâmetros $E_{a}$ e $\rho_{\infty}$.

\section{MATERIAIS E MÉTODOS}

Para a determinação da massa específica do leite de coco, utilizou-se a modelagem matemática obtida por Tansakul e Chaisawang (2006) e indicada pela Equação 4. Esses autores estudaram o efeito da temperatura e do teor de gordura sobre a massa específica do leite de coco, entre 60 e $80^{\circ} \mathrm{C}$ e o teor de gordura variando de 20 a $35 \%$.

$$
\rho=1018-31,7 \cdot X_{F}-0,465 \cdot T
$$

Em que $X_{F}($ em \%) corresponde ao teor de gordura e $T\left(\mathrm{em}^{\circ} \mathrm{C}\right)$, a temperatura.

Com base na Equação 4, foram estimados os valores da massa específica do leite de coco para as temperaturas de 60, 62, $65,68,70,72,75,78$ e $80^{\circ} \mathrm{C}$ e para os teores de gordura de 20, 25, 30 e $35 \%$.

A Tabela 1 relaciona os valores estimados da massa específica do leite de coco nos teores de gordura de 20 e $25 \%$, enquanto que a Tabela 2, os valores estimados da massa específica do leite de coco nos teores de gordura de 30 e $35 \%$. 
Tabela 1. Efeito da temperatura e do teor de gordura (20 e 25\%) sobre a massa específica do leite de coco

\begin{tabular}{ccc}
\hline $\begin{array}{c}\text { Temperatura } \\
\left({ }^{\circ} \mathrm{C}\right)(\mathrm{K})\end{array}$ & $\begin{array}{c}\text { Massa específica } \\
\left(\mathrm{kg} \cdot \mathrm{m}^{-3}\right)\end{array}$ & $\begin{array}{c}\text { Massa específica } \\
\left(\mathrm{kg} . \mathrm{m}^{-3}\right)\end{array}$ \\
& $\begin{array}{c}\text { Teor de gordura } \\
20 \%\end{array}$ & $\begin{array}{c}\text { Teor de gordura } \\
25 \%\end{array}$ \\
\hline $60(333,15)$ & 983,76 & 982,18 \\
$62(335,15)$ & 982,83 & 981,25 \\
$65(338,15)$ & 981,44 & 979,85 \\
$68(341,15)$ & 980,04 & 978,46 \\
$70(343,15)$ & 979,11 & 977,53 \\
$72(345,15)$ & 978,18 & 976,60 \\
$75(348,15)$ & 976,79 & 975,20 \\
$78(351,15)$ & 975,39 & 973,81 \\
$80(353,15)$ & 974,46 & 972,88 \\
\hline
\end{tabular}

Fonte: Autor.

Tabela 2. Efeito da temperatura e do teor de gordura (30 e 35\%) sobre a massa específica do leite de coco

\begin{tabular}{ccc}
\hline $\begin{array}{c}\text { Temperatura } \\
\left({ }^{\circ} \mathrm{C}\right)(\mathrm{K})\end{array}$ & $\begin{array}{c}\text { Massa específica } \\
\left(\mathrm{kg} . \mathrm{m}^{-3}\right)\end{array}$ & $\begin{array}{c}\text { Massa específica } \\
\left(\mathrm{kg} . \mathrm{m}^{-3}\right)\end{array}$ \\
& $\begin{array}{c}\text { Teor de gordura } \\
30 \%\end{array}$ & $\begin{array}{c}\text { Teor de gordura } \\
35 \%\end{array}$ \\
\hline $60(333,15)$ & 983,76 & 979,01 \\
$62(335,15)$ & 982,83 & 978,08 \\
$65(338,15)$ & 981,44 & 976,68 \\
$68(341,15)$ & 980,04 & 975,29 \\
$70(343,15)$ & 979,11 & 974,36 \\
$72(345,15)$ & 978,18 & 973,43 \\
$75(348,15)$ & 976,79 & 972,03 \\
$78(351,15)$ & 975,39 & 970,64 \\
$80(353,15)$ & 974,46 & 969,71 \\
\hline
\end{tabular}

Fonte: Autor.

As Equações 5 e 6 determinam, respectivamente, os valores de $\left(\frac{E_{a}}{R}\right)$ e $\ln \rho_{\infty}$, sendo adaptadas do trabalho de Triola (2008).

A Equação 7 determina o valor do coeficiente de correlação linear $\left(r^{2}\right)$ para a análise de regressão linear dos dados emparelhados de $\ln \rho$ e $\frac{1}{T}$. Essa equação também foi adaptada do trabalho de Triola (2008).

$$
\begin{gathered}
\frac{E_{a}}{R}=\frac{\left\{n \cdot\left[\sum_{i=1}^{n}\left(\frac{1}{T}\right) \cdot \ln \rho\right]-\left[\sum_{i=1}^{n}\left(\frac{1}{T}\right) \cdot \sum_{i=1}^{n} \ln \rho\right]\right\}}{\left\{n \cdot\left[\sum_{i=1}^{n}\left(\frac{1}{T}\right)^{2}\right]-\left[\sum_{i=1}^{n}\left(\frac{1}{T}\right)\right]^{2}\right\}} \\
r^{2}=\frac{\left\{\left(\sum_{i=1}^{n} \ln \rho\right)-\left(\frac{E_{a}}{R}\right) \cdot\left(\sum_{i=1}^{n} \frac{1}{T}\right)\right\}}{\left.\left\{\int\left[n \cdot \sum_{i=1}^{n}\left(\frac{1}{T}\right)^{2}\right]-\left[\sum_{i=1}^{n}\left(\frac{1}{T}\right)\right]^{2}\right\}^{1 / 2} \cdot\left\{\left[n \cdot \sum_{i=1}^{n}(\ln \rho)^{2}\right]-\left[\sum_{i=1}^{n} \ln \rho\right]^{2}\right\}^{1 / 2}\right\}}
\end{gathered}
$$

Com base nos dados indicados nas Tabelas 1 e 2, os valores da razão $\left(\frac{E_{a}}{R}\right)$ e $\ln \rho_{\infty}$ foram obtidos a partir da análise de regressão linear dos valores de $\ln \rho$ em função de $\frac{1}{T}$ para cada leite de coco com teor de gordura diferente, conforme indicados pelas Equações 5 e 6 .

Nas Equações 5, 6 e 7, $n$ corresponde ao número de dados emparelhados de $\ln \rho$ em função de $\frac{1}{T}$, que de acordo com as Tabelas 1 e 2, equivale a 9 .

Nos cálculos de $\frac{1}{T}$, a temperatura utilizada foi na escala graus Kelvin.

No cálculo para a determinação dos valores da energia de ativação para a massa específica $\left(E_{a}\right)$, considerou-se que o valor da constante universal dos gases ideais $(R)$ 
equivale a $8,314 \mathrm{~J} \cdot \mathrm{mol}^{-1} \cdot \mathrm{K}^{-1} \quad(\mathrm{NETZ}$ e ORTEGA, 2008).

\section{RESULTADOS E DISCUSSÃO}

A Figura 1 ilustra o gráfico de $\ln \rho$ versus $\frac{1}{T}$ para o leite de coco.

Figura 1: Gráfico de $\ln \rho$ versus $\frac{1}{T}$ para o leite de coco

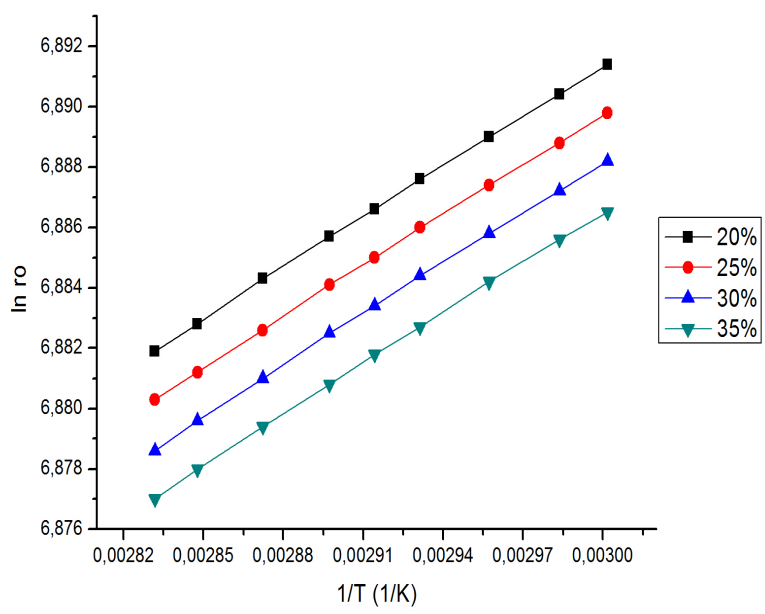

Fonte: Autor.

A Tabela 3 relaciona os resultados obtidos através das Equações 5, 6 e 7.

\begin{tabular}{cccc}
\multicolumn{3}{c}{ Tabela 3. Valores obtidos para $\frac{E_{a}}{R}, \ln \rho_{\infty}$ e $r^{2}$} \\
\hline Leite de coco & $\frac{E_{a}}{R}(\mathrm{~K})$ & $\ln \rho_{\infty}$ & $r^{2}$ \\
& & & \\
\hline $20 \%$ de gordura & 55,8038 & 6,7240 & 0,9998 \\
$25 \%$ de gordura & 55,9579 & 6,7219 & 0,9998 \\
$30 \%$ de gordura & 56,2576 & 6,7194 & 0,9998 \\
$35 \%$ de gordura & 55,9544 & 6,7187 & 0,9998 \\
\hline
\end{tabular}
Fonte: Autor.

$$
\text { Pinheiros e coautores }
$$

comentam que o coeficiente de correlação linear $\left(r^{2}\right)$ mede a interdependência linear entre as variáveis e avalia a qualidade do ajuste, ou seja, quanto mais próximo o coeficiente de correlação linear for da unidade, melhor o ajuste da reta em relação aos pontos da dispersão.

Pode-se observar na Tabela 3 que o coeficiente de correlação linear, para todos os teores de gordura do leite de coco estudados, está próximo da unidade.

Lira (2004) e Carvalho (2005) comentam que a correlação linear é classificada como muito forte quando os valores do coeficiente de correlação linear são maiores ou iguais a 0,90 e menores que 1,0 . Dessa forma, para todos os teores de gordura do leite de coco estudados, a correlação linear é classificada como muito forte.

A Tabela 4 relaciona os valores da energia de ativação para a massa específica $\left(E_{a}\right)$ e da massa específica quando a temperatura tende ao infinito $\left(\rho_{\infty}\right)$.

Tabela 4. Valores obtidos para $E_{a}$ e $\rho_{\infty}$

\begin{tabular}{ccc}
\hline Leite de coco & $E_{a}\left(\mathrm{~J} \cdot \mathrm{mol}^{-1}\right)$ & $\rho_{\infty}\left(\mathrm{kg} \cdot \mathrm{m}^{-3}\right)$ \\
\hline 20\% de gordura & 463,9528 & 832,1394 \\
25\% de gordura & 465,2340 & 830,3938 \\
30\% de gordura & 467,7257 & 828,3204 \\
35\% de gordura & 465,2049 & 827,7408 \\
\hline Fonte: Autor & &
\end{tabular}

Fonte: Autor

Considerando que a energia de ativação para a massa específica $\left(E_{a}\right)$ indica 
a sensibilidade da massa específica devido à variação da temperatura, os resultados sugerem que o leite de coco com $30 \%$ de gordura apresenta uma maior sensibilidade da massa específica em virtude da variação da temperatura, em comparação aos demais teores de gordura do leite de coco estudados.

Pode-se observar na Tabela 4, que com o aumento do teor de gordura, aumentase a energia de ativação para a massa específica $\left(E_{a}\right)$, entre teores de gordura de 20 a 30\%. Nos teores de gordura de 30 a 35\%, ocorre uma diminuição da energia de ativação para a massa específica.

A ausência na literatura de valores experimentais da energia de ativação para a massa específica $\left(E_{a}\right)$ e da massa específica quando a temperatura tende ao infinito $\left(\rho_{\infty}\right)$ do leite de coco em seus teores de gordura estudados dificultou a comparação com os valores encontrados nesse trabalho.

\section{CONCLUSÃO}

O objetivo desse trabalho foi avaliar o efeito da temperatura e do teor de gordura sobre a massa específica do leite de coco.

Os valores encontrados para a energia de ativação para a massa específica variaram de 463,9528 (teor de gordura de 20\%) a 467,7257 J.mol ${ }^{-1}$ (teor de gordura de 30\%), em um intervalo de temperatura entre 333,15 a $353,15 \mathrm{~K}$ e pressão de 1 atm.
Os resultados sugerem que com o aumento do teor de gordura, aumenta-se a energia de ativação para a massa específica, entre teores de gordura de 20 a 30\%. Nos teores de gordura de 30 a 35\%, ocorre uma diminuição da energia de ativação para a massa específica.

Considerando que a energia de ativação para a massa específica $\left(E_{a}\right)$ indica a sensibilidade da massa específica devido à variação da temperatura, os resultados sugerem que o leite de coco com $30 \%$ de gordura apresenta uma maior sensibilidade da massa específica em virtude da variação da temperatura, em comparação aos demais teores de gordura do leite de coco estudados.

$\mathrm{Na}$ literatura, não foram encontrados valores experimentais da energia de ativação para a massa específica do leite de coco, o que dificultou a comparação dos resultados.

\section{REFERÊNCIAS}

ALCÂNTARA， L.A.P.; FONTAN， R.C.I.; BONOMO, R.C.F.; LEMOS, A.R. Efeito da temperatura e teor de gordura nas propriedades termofísicas do leite de coco. Revista Brasileira de produtos Agroindustriais, v. 14, n. 1, p.21-30, 2012.

BROWN, L.S.; HOLME, T.A. Química geral aplicada à Engenharia. Cengage Learning, São Paulo, 2009. 653p. 
CANCIAM, C.A. Efeito da temperatura na massa específica de ésteres metílicos de ácidos graxos. Revista da Universidade Vale do Rio Verde, v. 12, n. 1, p.17-27, 2014.

CARVALHO, M.J.S. Quimiometrica e espectroscopia no infravermelho próximo (NIR) e médio (MIR) aplicadas na análise de óleos lubrificantes. Dissertação de Mestrado do Programa de Mestrado em Química do Instituto Militar de Engenharia, 2005. 121p.

FREUND, J. E. Estatística aplicada Economia, Administração e Contabilidade. Artmed, Porto Alegre, 2004. 545p.

GIAP, S. G. E. The hidden property of Arrhenius-type relationship: viscosity as a function of temperature. Journal of Physical Science, v. 2, n. 1, p. 29-39, 2010.

LIRA, S. A. Análise de correlação: abordagem teórica e de construção dos coeficientes com aplicação. Dissertação de Mestrado do Programa de Mestrado em Ciências da Universidade Federal do Paraná, 2004. 196p.

MAGERRAMOV, M.A.; ABDULAGATOV, A.I.; AZIZOV, N.D.; ABDULAGATOV, I.M. Pressure- and temperature-dependent density change of juices during concentration. Food Bioprocess Technology, n.1, p.254-269, 2008.
MATOS, M.A.A. Propriedades termofísicas de alguns gases, sólidos e da água. Editora da Universidade de Aveiro, Aveiro, 1998. 30p.

MORRISON, R.T.; BOYD, R.N. Química Orgânica. Fundação Calouste Gulbekian, Lisboa, 1996. 1394p.

NETZ，P.A.; ORTEGA，G.G. Fundamentos de físico-química: uma abordagem conceitual para as ciências farmacêuticas. Artmed, Porto Alegre, 2008. 299p.

PINHEIROS, J.I.D.; CUNHA, S.B.; CARVAJAL, S.R.; GOMES, G.C. Estatística básica: a arte de trabalhar com dados. Elsevier, Rio de Janeiro, 2009. 295p.

SCHONS, E.M. Desestabilização de emulsões visando a redução do teor de óleo em água. Dissertação de Mestrado do programa de Mestrado em Engenharia Mineral da Universidade Federal de Ouro Preto, 2008. $168 p$.

TANSAKUL, A.; CHAISAWANG, P. Thermophysical properties of coconut milk. Journal of Food Engineering, n. 73, p.276280, 2006.

TEIXEIRA， E.A.M.; MAIA， G.A.; HOLANDA， L.F.F.; OLIVEIRA， G.S.F.; GASPAR JÚNIOR, J.C.; FIGEUIREDO, R.W. Processo alternativo para conservação 
do leite de coco produzido para consumo comercial. Revista Pesquisa Agropecuária Brasileira, v. 24, n. 6, p.761-768, 1989.

TEIXEIRA， V.G.; COUTINHO, F.M.B.; GOMES, A.S. Principais métodos de caracterização de resinas à base de divinilbenzeno. Química Nova, v. 24, n. 6, p.808-818, 2001.

TRIOLA, M.F. Introdução à Estatística. LTC, Rio de Janeiro, 2008. 720p.

TSEN, J.H.; KING, V.A.E. Density of banana puree as a function of soluble solids concentration and temperature. Journal of Food Engineering, v. 55, p.305-308, 2002. 